\title{
Livello di dipendenza dai Servizi Territoriali e costi relativi al trattamento della schizofrenia
}

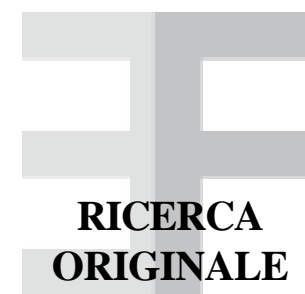

Giorgio D’Allio*, Fernando Rutto*, Michela Coppi*, Luca Guidi§

\begin{abstract}
The present study was designed to evaluate the level of dependence from Mental Health Care Department, in Casale Monferrato, of three groups of psychotic patients treated with olanzapine (31), risperidone (30) or typical neuroleptics (31). The observation was retrospective, lasting one year (2003-2004), and collected data relative to health care resources as specialist visits, home interventions operated by nurses or physicians, drug administration, rehabilitation, psychotherapy, hospitalizations. The data collected allowed to evidentiate substantial differences among olanzapine and risperidone treated patients, usually younger, versus typical treated patients, usually older and more chronic. In general, atypical treated patients, evidentiate a reduction of home nurse intervention in respect to typical treated patients while olanzapine shows a trend in hospitalization and specialist visits reduction versus risperidone. Total health care costs are not significantly different among the three groups but evidentiate interventions more oriented to rehabilitation in the group treated with olanzapine while risperidone treated patients needed a major number of hospitalizations. Typical treated patients requested, instead, an high number of home intervention due to their chronic conditions and cognitive imparement.
\end{abstract}

Keywords: costs, olanzapine, risperidone, typical neuroleptics, psychosis

Farmeconomia e percorsi terapeutici 2005; 6 (4): 301-304

\section{INTRODUZIONE}

Gli antipsicotici atipici hanno dimostrato con numerose pubblicazioni [3-15] di offrire, rispetto ai neurolettici tipici, molti vantaggi in termini di ottimizzazione nell'impiego delle risorse sanitarie usate per la gestione delle psicosi. In particolare numerosi autori $[3,4,5,6,8,12]$ hanno verificato che il trattamento con olanzapina permette, in associazione ad adeguate attività riabilitative, di rendere $\mathrm{i}$ pazienti meno dipendenti dai Servizi Sanitari con conseguente riduzione della richiesta di interventi medici, infermieristici e di supporto sociale $[3,4,5,13,15]$. A tale riduzione della domanda/necessità di servizio corrisponde un risparmio economico significativo, sufficiente a compensare del tutto o in parte i maggiori costi di acquisizione dei nuovi farmaci $[2,3,15]$. Oltre a questi aspetti gestionali, non possono essere trascurati i benefici per la qualità della vita dei pazienti e delle loro famiglie $[6,8]$ conseguenti direttamente da una conduzione meno incisiva e, in definitiva cruenta, del rapporto terapeutico. In alcune precedenti ricerche, alla riduzione del numero degli interventi territoriali e ospedalieri è corrisposto anche un in- cremento della partecipazione lavorativa e di studio dei pazienti [15].

Dati questi presupposti, confermati già in alcune realtà italiane $[3,4,15]$, con il nostro studio abbiamo voluto valutare il livello di dipendenza dai Servizi Psichiatrici di tre gruppi di pazienti seguiti dal Dipartimento di Salute Mentale e delle Dipendenze (DSMD) di Casale Monferrato e in trattamento con antipsicotici atipici (olanzapina e risperidone) o aloperidolo durante il 2003/2004.

\section{MATERIALIEMETODI}

Il punto di vista adottato per questa analisi è quello della ASL, e in particolare del DSMD. Sono stati arruolati, retrospettivamente, 92 pazienti schizofrenici seguiti durante il 2003 e il 2004 presso il DSMD di Casale Monferrato e trattati con olanzapina (OLZ) (31 pazienti), risperidone (RIS) (30 pazienti) e neurolettici tipici (NL) (31 pazienti). L'assegnazione dei pazienti ai tre gruppi (OLZ, RIS e NL) è avvenuta considerando come principale il farmaco antipsicotico o neurolettico assunto a dosi piene per i primi 7 mesi dell'osservazione. Si è cerca-
* Servizio di Psichiatria, Dipartimento di Salute Mentale e delle Dipendenze (DSMD), ASL 21, Casale Monferrato (AL)

$\S$ Eli Lilly Italia S.p.A., Sesto Fiorentino (FI) 


\begin{tabular}{|c|c|c|c|c|c|c|}
\hline & OLZ & RIS & ALO & OLZ vs RIS & OLZ vs ALO & RIS vs ALO \\
\hline N gg Servizio diurno & 0 & 0 & 0 & ns & ns & ns \\
\hline $\mathrm{N}$ visite specialistiche & 12,6 & 13,8 & 8,1 & ns & 0,012 & 0,006 \\
\hline $\mathrm{N}$ interventi infermieristici in struttura & 0 & 0 & 0 & ns & ns & ns \\
\hline $\mathrm{N}$ interventi infermieristici domiciliari & 6,1 & 8,9 & 18,3 & ns & 0 & 0 \\
\hline $\mathrm{N}$ interventi medici domiciliari & 0,2 & 0 & 0 & ns & ns & ns \\
\hline $\mathrm{N}$ interventi di psicoterapia & 0,1 & 0 & 0,3 & ns & ns & 0,036 \\
\hline $\mathrm{N}$ interventi di riabilitazione & 0,1 & 1,3 & 0 & ns & ns & ns \\
\hline N. di ricoveri & 0,1 & 0,2 & 0,1 & ns & ns & ns \\
\hline N gg di ricovero & 1,5 & 2,1 & 1,3 & ns & ns & $n$ \\
\hline
\end{tabular}

\section{Tabella I}

Numero medio di interventi sanitari suddivisi per tipologia e terapia somministrata

$\mathrm{OLZ}=$ olanzapina; $\mathrm{RIS}=$ risperidone; $\mathrm{ALO}=$ aloperidolo

to di avere una sufficiente omogeneità tra i gruppi di pazienti a confronto basandosi sul numero di ricoveri nell'anno precedente e sulla conoscenza diretta da parte dei clinici. Si è in tal modo riusciti a costruire gruppi sufficientemente confrontabili per olanzapina e risperidone, composti prevalentemente da pazienti di recente diagnosi, mentre il gruppo di trattamento con aloperidolo è risultato essere significativamente diverso. In particolare questo gruppo era costituito esclusivamente da pazienti con lunga storia di malattia e trattamento che avevano raggiunto una buona stabilità, mantenuta a dosi molto basse di aloperidolo.

I dati relativi agli interventi sanitari (ricoveri e assistenza sul territorio) operati sui pazienti nel corso del 2003 sono stati estratti dalle relative cartelle cliniche.

In coerenza con il punto di vista adottato per la nostra analisi, abbiamo effettuato l'attri-

\begin{tabular}{lccc}
\hline & OLZ & RIS & ALO \\
\hline Servizio diurno & 0 & 0 & 0 \\
Visite specialistiche & 640,78 & 702,79 & 409,71 \\
Interventi infermieristici in struttura & 0 & 0,29 & 0 \\
Interventi infermieristici domiciliari & 152,47 & 223,76 & 459,85 \\
Interventi medici domiciliari & 12,74 & 0 & 0 \\
Interventi di psicoterapia & 4,3 & 0 & 19,37 \\
Interventi di riabilitazione & 3,84 & 51,53 & 0 \\
Giornate di ricovero & 232,71 & 502,17 & 316,27 \\
TOTALE & $1.046,84$ & $1.480,54$ & $1.205,10$ \\
\hline
\end{tabular}

Tabella II

Costi medi per gli interventi sanitari (in euro)

$\mathrm{OLZ}=$ olanzapina; $\mathrm{RIS}=$ risperidone; $\mathrm{ALO}=$ aloperidolo buzione dei costi riferendosi a quelli reali di erogazione delle singole prestazioni, pubblicati con i risultati dello studio HoNOS 2 [2], e non quelli indicati dai Tariffari o Nomenclatori Regionali.

\section{RISULTATI}

Obiettivo della studio era valutare il livello di dipendenza dai Servizi Sanitari di Territorio e Ospedalieri di tre diversi gruppi di pazienti affetti da schizofrenia e trattati con olanzapina, risperidone e aloperidolo.

A fronte di una buona confrontabilità per caratteristiche cliniche dei pazienti in trattamento durante l'osservazione con olanzapina e risperidone, si è dovuta rilevare una importante differenza tra questi due gruppi e quello trattato con aloperidolo. Questo gruppo era infatti costituito da pazienti di vecchia diagnosi ormai stabilizzati con una terapia a bassi dosaggi e senza associazione di altri principi attivi.

In Tabella I vengono presentate le frequenze per i principali interventi sanitari operati sul nostro campione.

In generale si evidenzia che gli atipici hanno ridotto significativamente il numero di interventi degli infermieri presso il domicilio dei pazienti, pur richiedendo però un maggior numero di visite specialistiche. Nessun paziente, nei tre gruppi, si è riferito ad un Centro Diurno. Sebbene non sia stata raggiunta la significatività, i pazienti in trattamento con risperidone hanno fatto registrare un tasso di ricovero, per numero di ricoveri e per loro durata, tendenzialmente superiore a quello di olanzapina e aloperidolo. È risultato trascurabile il ricorso ai servizi infermieristici in struttura, alla psicoterapia (verificatosi solo nel gruppo trattato con aloperidolo) e alla riabilitazione. 
Assegnando a ciascun intervento sanitario il relativo costo di erogazione tratto dai risultati dello studio HoNOS 2 [2)] si ottiene il costo medico diretto medio per l'assistenza del nostro campione di pazienti (Tabella II).

I costi totali non sono significativamente differenti, ma evidenziano una riduzione di domanda di servizi sanitari maggiore per i pazienti in trattamento con olanzapina rispetto a quelli trattati con aloperidolo e, in particolare, rispetto a quelli trattati con risperidone.

Le differenze maggiori sono a carico degli interventi domiciliari infermieristici, particolarmente accentuati tra $\mathrm{i}$ pazienti trattati con aloperidolo, e i ricoveri e le visite specialistiche, maggiormente numerosi tra i pazienti trattati con risperidone (Figura 1).

Da evidenziare il fatto che si sia verificata una quasi assenza di ricorso alle attività riabilitative in tutti e tre $\mathrm{i}$ gruppi.

\section{DISCUSSIONE}

Il presente studio ha tra i suoi limiti quello di non poter testimoniare l'evoluzione clinica dei pazienti nell'anno di osservazione a causa della mancata rilevazione con strumenti strutturati, quali le scale di valutazione psichiatrica, nel periodo di osservazione. Questa carenza è stata superata, come ormai consolidato, assumendo come proxy di gravità media dei gruppi il numero di ricoveri nell'anno precedente all'osservazione. I tre campioni di pazienti osservati avevano tutti, nell'anno precedente, lo stesso numero e durata di ricoveri. Nonostante ciò, questo criterio non è stato sufficiente a garantire una buona confrontabilità tra i pazienti trattati con gli atipici, olanzapina e risperidone, e quelli trattati con aloperidolo. I pazienti trattati con aloperidolo dimostravano tutti una maggior anzianità di malattia e una maggior stabilizzazione, dimostrata dal mantenimento effettuato a dosi significativamente inferiori di quella terapeutica.

Considerando il diverso ricorso nei tre gruppi alle risorse sanitarie, si possono identificare altrettanti percorsi di cura prevalenti consolidati nella pratica del DSM. In particolare i pazienti trattati con aloperidolo, ricorrendo maggiormente all' assistenza domiciliare, dimostrano la necessità di un costante supporto per l'assunzione della terapia e una ridotta indipendenza funzionale. Diversamente i pazienti che assumono gli atipici presentano un maggior ricorso alle visite specialistiche, dovuto alla relativamente minor anzianità di cura ma un basso livello di domanda di interventi domiciliari, facendo ritenere di essere più indipendenti dal punto di vista dell'assunzione dei farmaci.

I ricoveri, pur non evidenziando differenze significative tra i gruppi, depongono per una maggior efficacia di intervento nei gruppi di

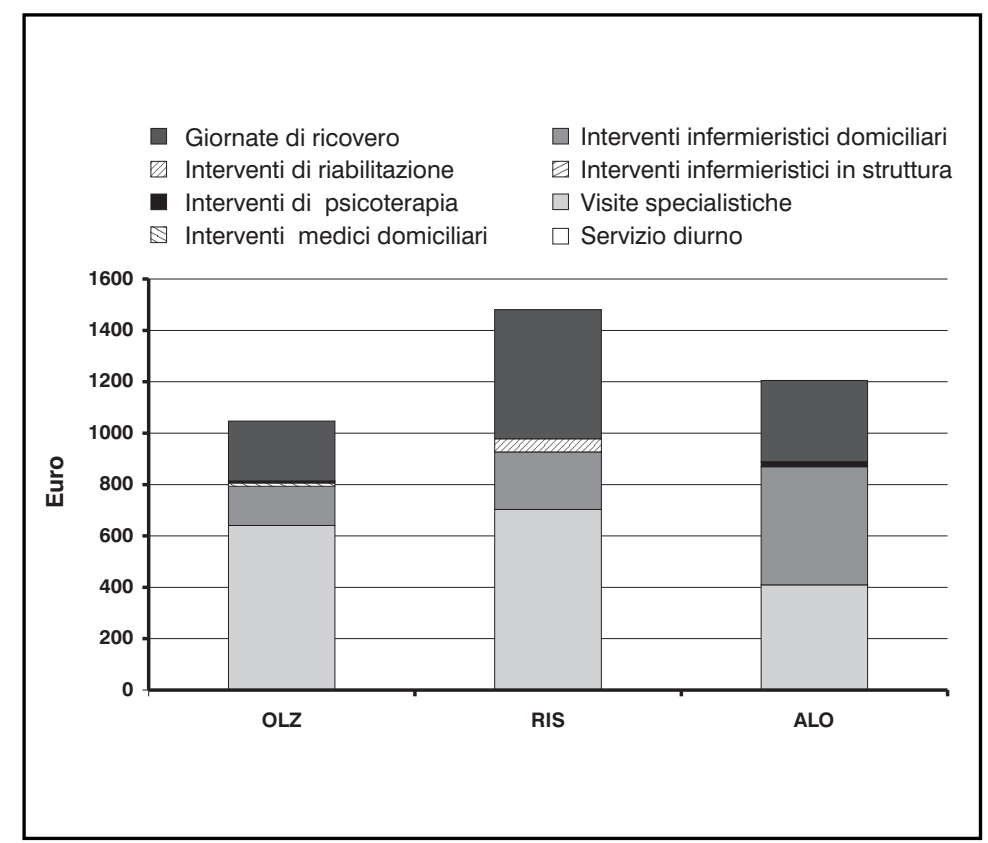

Figura 1

Costo medio a paziente per gli interventi sanitari

trattamento olanzapina e aloperidolo dove si riducono sia numerosità che durata delle ospedalizzazioni.

Considerando nel complesso la pressione esercitata dai tre gruppi osservati sui Servizi Psichiatrici del DSM di Casale Monferrato, si può evidenziare che tendenzialmente il miglior assetto di intervento sanitario sembra essere quello assunto con il gruppo di trattamento olanzapina. Tale gruppo infatti ricorre meno ai ricoveri e all'assistenza domiciliare permettendo una riduzione dei costi di erogazione delle prestazioni e una maggior continuità di cura per i pazienti. Anche il gruppo di trattamento aloperidolo ha evidenziato un basso ricorso ai ricoveri, testimoniando una sufficiente efficacia di intervento, ma ha richiesto una quantità significativa di assistenza domiciliare. I pazienti di questo gruppo sono quelli con una maggior durata di cura ed una relativa stabilità di malattia. Il ricorso agli interventi domiciliari si rende necessario per garantire l' assunzione della terapia e mantenere bassa la probabilità di ricaduta e quindi di ricovero. Il gruppo che assumeva risperidone, invece, non sembra aver trovato nel suo percorso di cura un equilibrio. Il numero di visite specialistiche è ancora il più elevato ma non sufficiente come intervento per ridurre il numero e la durata dei ricoveri.

In conclusione il percorso seguito dai pazienti in trattamento con olanzapina appare tendenzialmente il più equilibrato tra esigenze di contenimento dei costi di erogazione delle prestazioni sanitarie ed il raggiungimento di una relativa indipendenza dei pazienti sia da forme croniche di assistenza alla persona che da interventi in urgenza quali i ricoveri. 


\section{BIBLIOGRAFIA}

1. Garattini L, Zanelli E, Vesconi D. Analisi dei costi per divisione su un campione di nove presidi ospedalieri. ASI 1996; 10:33-37.

2. Mapelli $\mathrm{V}$ et al. Pattern di trattamento e costi nei dipartimenti di salute mentale della regione lombardia. Il progetto di ricerca HONOS2. Epidemiologia e Psichiatria Sociale. 2002, supplemento

3. Rossi I et al. Valutazione dei costi di trattamento dei disturbi psicotici con olanzapina, risperidone e neurolettici tipici in un DSM Italiano. Farmacoeconomia e Percorsi Terapeutici, 2001, 2(4): 253-258;

4. Berardi D, Dell' Atti M, Guidi L, Russo F, Vagnini V. Costo del trattamento con olanzapina nei Servizi di Salute Mentale. Atti Congresso SIP 2000, pag. 820.

5. Hamilton SH et al. Functional outcomes in schizophrenia: a comparison od olanzapine and haloperidol in an European sample. Int. Clin. Psychopharmacology 2000; 15(5): 245-255.

6. Revicki DA, Genduso LA, Hamilton SH, et al. Olanzapine versus haloperidol in the treatment of schizophrenia and other related psychotic disorders: quality of life and clinical outcomes of a randomized clinical trial. Quality of Life Research 1999; 8:417-426.

7. Tran PV, Delva MA, Tollefson GD, et al. Extrapyramidal symptoms and tolerability of olanzapine versus haloperidol in the acute treatment of schizophrenia. Journal of Clinical Psychiatry 1997;58:205-11.

8. Grainger D. et al. Resource use and quality of life of olanzapine compared with risperidone: results from an international randomized clinical trial. European Neuropsychopharmacology 1998; 8(suppl.A): s225-s226.

9. Edgell ET et al. Olanzapine vs risperidone, a perspective comparison of clinical and economic outcomes in schizophrenia. Pharmacoeconomics 2000; 18(6): 567-579.

10. Sanger TM et al. Olanzapine vs haloperidol treatment in first-episode psychosis. Am. J. Psychiatry, 1999, 156(1): 7987.

11. Sacristan JA, Gomez JC, Carulla S. Analisis coste-efectividad de olanzapina frente a haloperidol en el trattamiento de la esquizofrenia en Espagna. Actas Luso-Esp Neurol. Psiquiatr., 1997, 25(4): 225-234.

12. Sacristan JA, Gomez JC, Martin J et al. Pharmacoeconomic assessment of olanzapine in the treatment of refractory schizophrenia based on a pilot clinical study. Clin. Drug Invest., 1998, 15(1):29-35.

13. Foster RH, Goa KL. Olanzapine. A pharmacoeconomic review of its use in schizophrenia. Pharmacoeconomics, 1999, 15(6): 611-640.

14. Almond S, O'Donnell O. Cost analysis of the treatment of schizophrenia in the UK, a comparison of olanzapine and haloperidol. Pharmacoeconomics, 1998, 13(5 pt 2): 575-588.

15. Barioglio A, Guidi L, Mariani G. Influenza del trattamento della schizofrenia con neurolettici tipici o olanzapina sui costi sanitari e sugli outcomes lavorativi. Farmeconomia e percorsi terapeutici, 2005, 6(2):1-4 